\title{
PNH clone assessment by flow cytometry and its clinical correlation in PNH and aplastic anemia
}

\author{
Sanjeev Kumar Gupta $\cdot$ Hara Prasad Pati • \\ Anusha Priyadarsini Tejomurtula • Tulika Seth
}

Received: 9 September 2010 / Accepted: 10 November 2010/Published online: 23 November 2010

(C) Springer-Verlag 2010

\begin{abstract}
Flow cytometry is the most sensitive and specific diagnostic modality for paroxysmal nocturnal hemoglobinuria (PNH) clone assessment in PNH and other bone marrow failure states. A total of 101 samples included $23 \mathrm{PNH}, 46$ aplastic anemia (AA), seven myelodysplastic syndrome (MDS) cases, and 25 normal controls. Flow cytometry was performed using CD55, CD59, and stain-lyse-wash method, and the PNH clone size was correlated with the severity of disease. The PNH clone size on granulocytes in $\mathrm{PNH}$ patients varied from $7 \%$ to $97 \%$ detected with a sensitivity of $0.2 \%$ and correlated with the clone on monocytes $(r=0.563 ; p<0.01)$. In PNH patients, a significant correlation was seen between PNH clone and the hemoglobin of patient $(r=-0.523 ; p<$ 0.05 ), however, not with the transfusion frequency, cytopenias, and hemolytic parameters $(p>0.05)$. PNH clone $(0.4$ $38.7 \%)$ was detected in $23(50 \%)$ AA patients. Three $(42.9 \%)$ of the seven MDS patients showed PNH clone $(<1 \%)$. CD55 and CD59 have the advantage of simultaneously analyzing granulocytes and monocytes for $\mathrm{PNH}$ clone which correlated with degree of anemia in PNH. A larger patient data is required to assess the clinical impact of PNH clone of variable size in AA and MDS patients.
\end{abstract}

Keywords PNH clone $\cdot$ Flow cytometry $\cdot \mathrm{PNH} \cdot$ Aplastic anemia $\cdot$ MDS

S. K. Gupta $\cdot$ H. P. Pati · A. P. Tejomurtula $\cdot$ T. Seth

Department of Haematology,

All India Institute of Medical Sciences,

IRCH Building, 1st floor, Ansari Nagar,

Delhi 110029, India

S. K. Gupta $(\bowtie)$

B-1/226, Yamuna Vihar,

Delhi 110053, India

e-mail: drskgupta1@gmail.com

\section{Introduction}

Paroxysmal nocturnal hemoglobinuria (PNH) is a rare acquired stem cell disorder characterized by deficiency of membrane-bound GPI (glycosylphosphatidylinositol)anchored proteins due to phosphatidylinositol glycan (PIG-A; class A) gene mutation. The clinical features of PNH include hemolysis with acute exacerbation, cytopenias of varying severity, and a tendency for thrombosis. Flow cytometry is the most sensitive and specific diagnostic modality for PNH assessment. Minor PNH clones have also been described in other bone marrow failure syndromes like aplastic anemia (AA), myelodysplastic syndrome (MDS), etc. This study is regarding the assessment of the PNH clone using flow cytometry in patients of $\mathrm{PNH}$, aplastic anemia, and MDS along with its clinical correlation, particularly in $\mathrm{PNH}$ patients.

\section{Materials and methods}

The present study was conducted in the Department of Hematology, All India Institute of Medical Sciences, New Delhi, India from November 2008 to December 2009. A total of 101 samples were studied which included 23 known cases of PNH (newly diagnosed as well as on follow-up), 46 patients of aplastic anemia, seven patients with MDS, and 25 normal controls.

A detailed clinical history including transfusion frequency was taken. A complete hemogram, reticulocyte count, plasma hemoglobin, lactate dehydrogenase (LDH), bone marrow examination, and urine for hemosiderin were done in PNH cases. All PNH patients had a cellular bone marrow with normal cellular morphology at the time of study. The 
aplastic anemia group had patients with bone marrow cellularity $<10 \%$. Twenty-five normal healthy controls (with no evidence of hemolysis, cytopenias, or thrombosis) were studied to set the detection range for flow cytometry test for $\mathrm{PNH}$ clone (above mean $+2 \mathrm{SD}$ value of normal controls). The institute's ethics committee approval was obtained for the project.

Flow cytometry The PNH clone size on granulocytes and monocytes was estimated by flow cytometry using peripheral blood sample collected in EDTA. Fluorescent-labeled mouse anti-human immunoglobulins CD55 FITC, CD59 $\mathrm{PE}$, and CD45 PE-Cy5 were used. All samples were subjected to flow cytometry within $4 \mathrm{~h}$ of collection by using the "stain-lyse-wash" method as described earlier [1]. Fifteen microliters of whole blood was taken with titrated amounts of CD 55FITC, CD 59PE, and CD45 PECy5. In cases of low absolute neutrophil count $($ ANC) $<1 \times$ $10^{9} / \mathrm{L}$, the blood sample was centrifuged at $1,000 \mathrm{rpm}$ for $5 \mathrm{~min}$, and the pipetting done to include the buffy coat to increase the yield of granulocytes. At least 20,000 events were acquired using Becton Dickinson FACS Canto flow cytometer and FACS DIVA software. The granulocytes and monocytes were identified by their characteristic properties on CD45/SSC dot-plot scattergrams and evaluated for expression of CD55 and CD59. Quadrant gates were applied on CD55/CD59 plot, and the percentage of cells negative for both antigens was calculated. The instrument set up was done on weekly basis for voltage and compensation settings using BD FACS seven color setup beads.

Clinical correlation The PNH clone size on granulocytes was correlated with the severity of disease (hemoglobin levels, transfusion frequency, cytopenias, and hemolytic parameters).

Data analysis Statistical analysis was done using SPSS for Windows version 15 (SPSS, Lead technology, 2006). Spearman's rank correlation was calculated. The comparison between AA patients with and without PNH clone was done by applying Mann-Whitney $U$ test. $p<0.05$ was considered as statistically significant.

\section{Observations and results}

Normal controls

We had 25 normal healthy controls including 14 males (M) and 11 females $(\mathrm{F})$ with median age of $28(17-45)$ years. Their median hemoglobin (Hb) was $14.4(12-16.4) \mathrm{g} / \mathrm{dL}$.
The granulocytes in the quadrant negative for both CD55 and CD59 ranged from $0 \%$ to $0.2 \%$ (median 0 ; mean 0.03 ; SD 0.063 ; Fig. 1). Thus, the PNH clone on granulocytes was defined as any value $>0.2 \%$ (mean $+2 \mathrm{SD}$ of normal controls). Similarly, this value for monocytes was $>0.7 \%$ (mean + 2SD of normal controls). The monocytes in the quadrant negative for both CD55 and CD59 ranged from $0 \%$ to $0.7 \%$ (median 0 ; mean 0.126 ; SD 0.189 ).

\section{PNH cases}

The $23 \mathrm{PNH}$ cases included $17 \mathrm{M}$ and $6 \mathrm{~F}$ with median age of 30 (12-55)years. The common signs and symptoms noted in PNH patients were pallor $(91.3 \%)$, bleeding (43.5\%), cola-color urine $(30.4 \%)$, icterus $(26.1 \%)$, and fever $(26.1 \%)$. Their median $\mathrm{Hb}$ was $8.7(4-12.8) \mathrm{g} / \mathrm{dL}$, total leukocyte count (TLC) $3.7(1.7-7.7) \times 10^{9} / \mathrm{L}$, ANC 1.7 $(0.6-5.2) \times 10^{9} / \mathrm{L}$, and platelet count $102(21-266) \times 10^{9} / \mathrm{L}$. The median reticulocyte count was $6.3 \%(3-32 \%)$, plasma hemoglobin $6.9(5.6-16.3) \mathrm{mg} / \mathrm{dL}$ (normal $<4 \mathrm{mg} / \mathrm{dL}$ ), and LDH 540 (350-3940) U/mL (normal adult range 240 $480 \mathrm{U} / \mathrm{mL}$ ). Urine for hemosiderin was positive in 12 out of $23(52.2 \%)$ patients. Two (8.6\%) patients developed thrombosis: one with DVT in left leg and the other in right middle cerebral artery after 4 and 5 years of PNH diagnosis, respectively.

Six out of 23 (26.1\%) patients had antecedent history of aplastic anemia. These included five males and one female patient ranging from 12 to 50 years of age. The duration between diagnosis of AA and PNH was 1 to 5 years. Five of these patients developed hemolytic PNH whereas one developed deep vein thrombosis (DVT; left leg). A 15-year male AA patient had responded to anti-thymocyte globulin 2 years back and then relapsed as hemolytic PNH.

On flow cytometry, the PNH clone size (on granulocytes) in PNH patients varied from $7 \%$ to $97 \%$ (Fig. 2). Sixteen $(69.6 \%)$ patients had $>50 \%$ clone size, six $(26.1 \%)$ had $10-50 \%$, and one patient had $<10 \%$ clone size. The PNH clone size on monocytes varied from $2.8 \%$ to $92.2 \%$ in these patients. The Spearman's rank correlation between PNH clone size on granulocytes and monocytes was highly significant $(r=0.563 ; p<0.01)$.

There was a significant negative correlation between $\mathrm{PNH}$ clone size on granulocytes and the $\mathrm{Hb}$ of patient $(r=-0.523 ; p<0.05$; Fig. 3$)$. However, the correlation of clone size on monocytes with the $\mathrm{Hb}$ was not statistically significant.

The median red cell transfusion frequency in $\mathrm{PNH}$ patients with $>50 \%$ clone size $(n=16)$ was 4 months ( 15 days to 15 months). PNH patients with $10-50 \%$ clone size $(n=6)$ received transfusion at a median of 6 months (1-13 months), and one patient with $<10 \%$ clone size required monthly transfusions (Table 1). 
Fig. 1 Gating of granulocytes (red color; P1) and monocytes (green color; $\mathrm{P} 2$ ) in the CD45/ SSC plot in normal control $(0 \%$ granulocytes in negative quadrant Q3; 0.1\% monocytes in Q3-1)

Fig. 2 PNH patient with large $\mathrm{PNH}$ clone on granulocytes (red color P $1 ; 88.7 \%$ in $\mathrm{Q} 3$ ) and monocytes (green color; P2; $92.2 \%$ in Q3-1)
NORMAL 15/9/09-STAINED

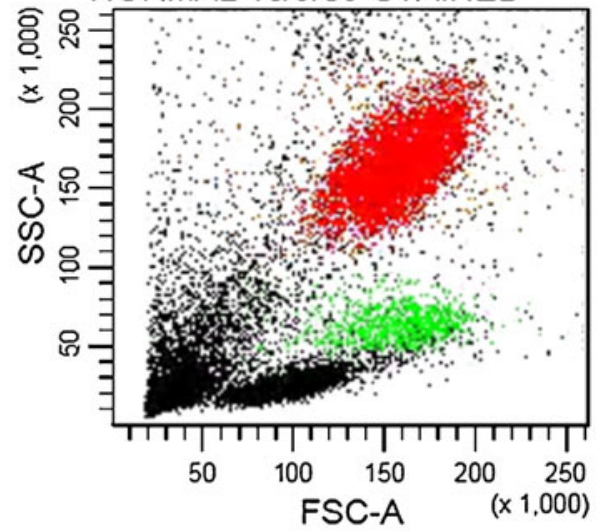

NORMAL 15/9/09-STAINED
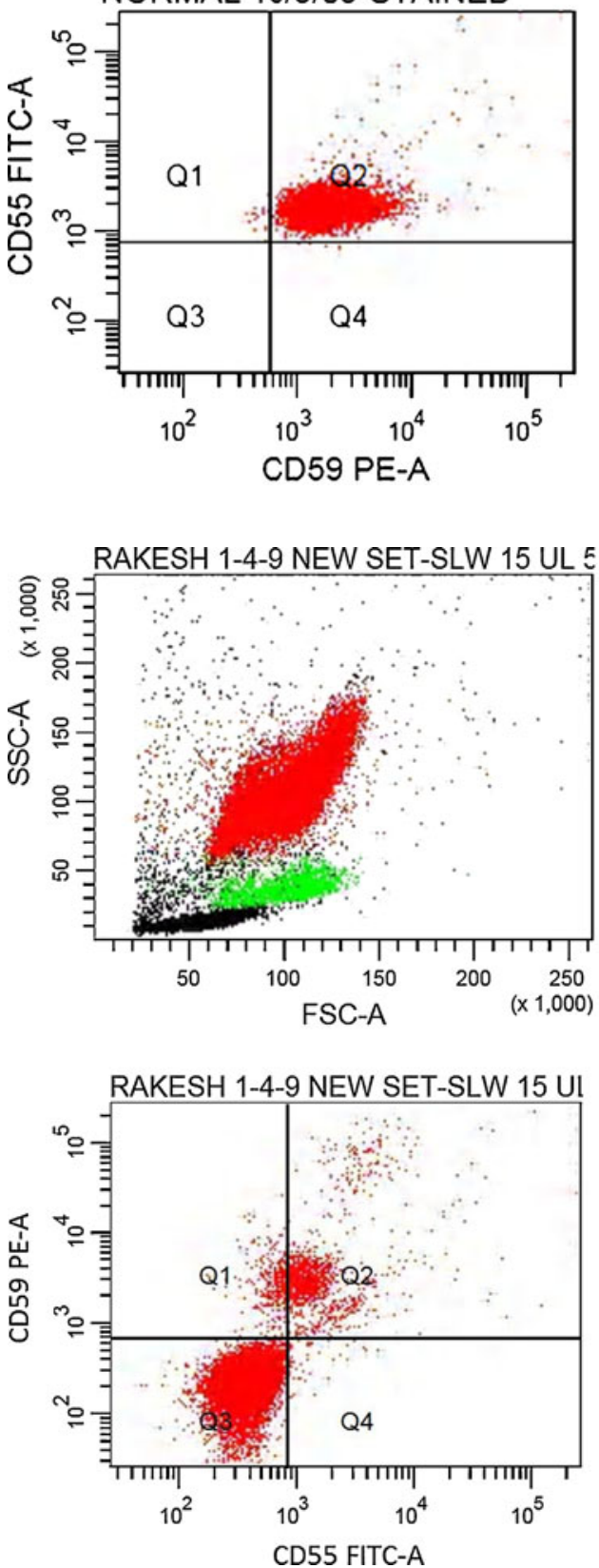

NORMAL 15/9/09-STAINED
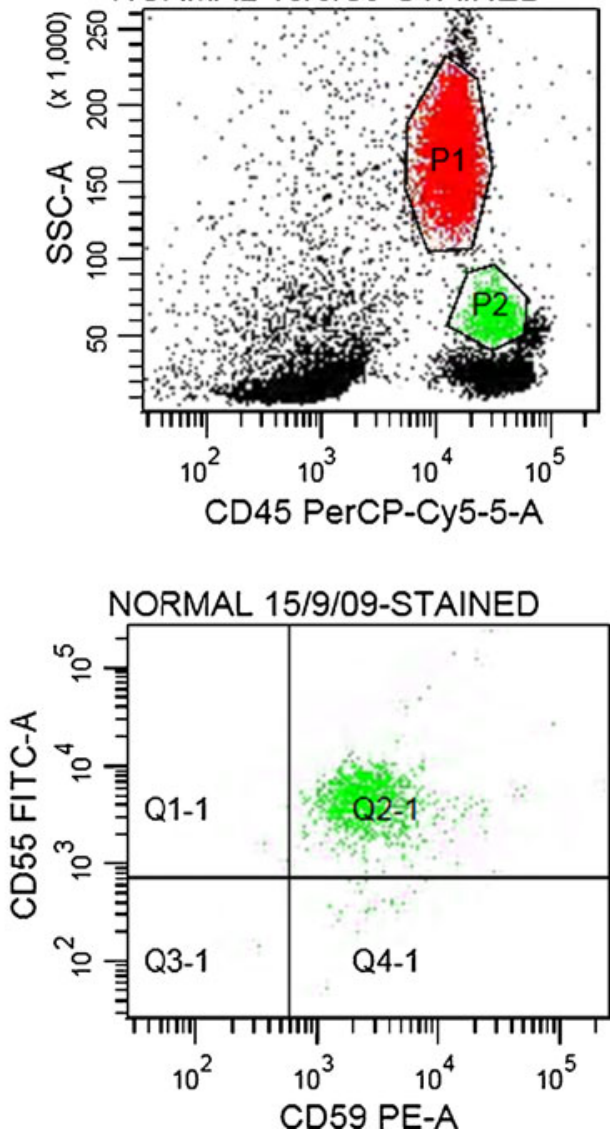

RAKESH 1-4-9 NEW SET-SLW $15 \mathrm{~L}$
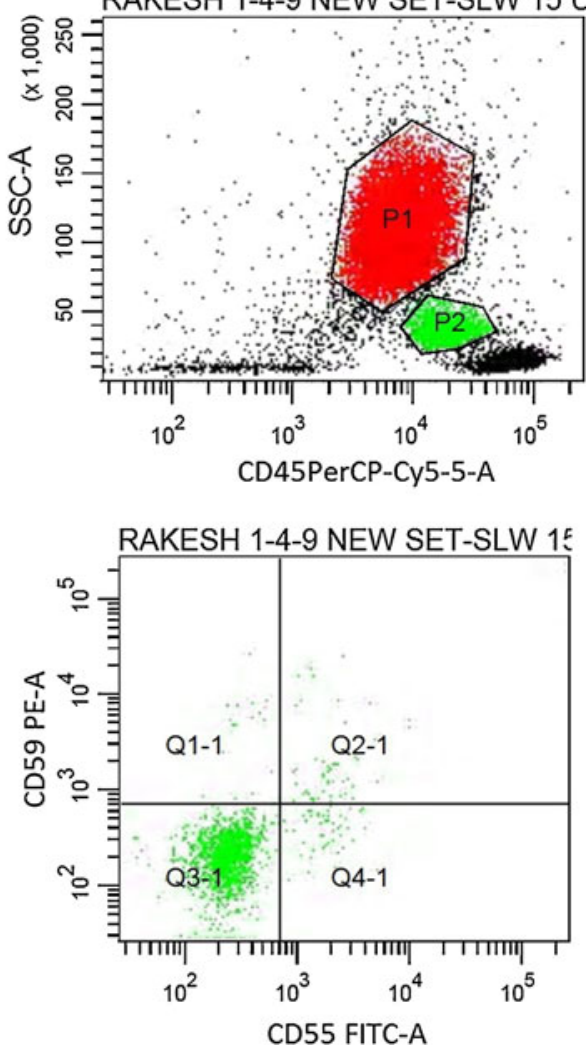
Fig. 3 Scatter plot between hemoglobin $(H b)$ and $\mathrm{PNH}$ clone on granulocytes in 23 PNH patients $(X$-axis- $\mathrm{PNH}$ clone on granulocytes; $\mathrm{Y}$-axis- $\mathrm{Hb}$ )

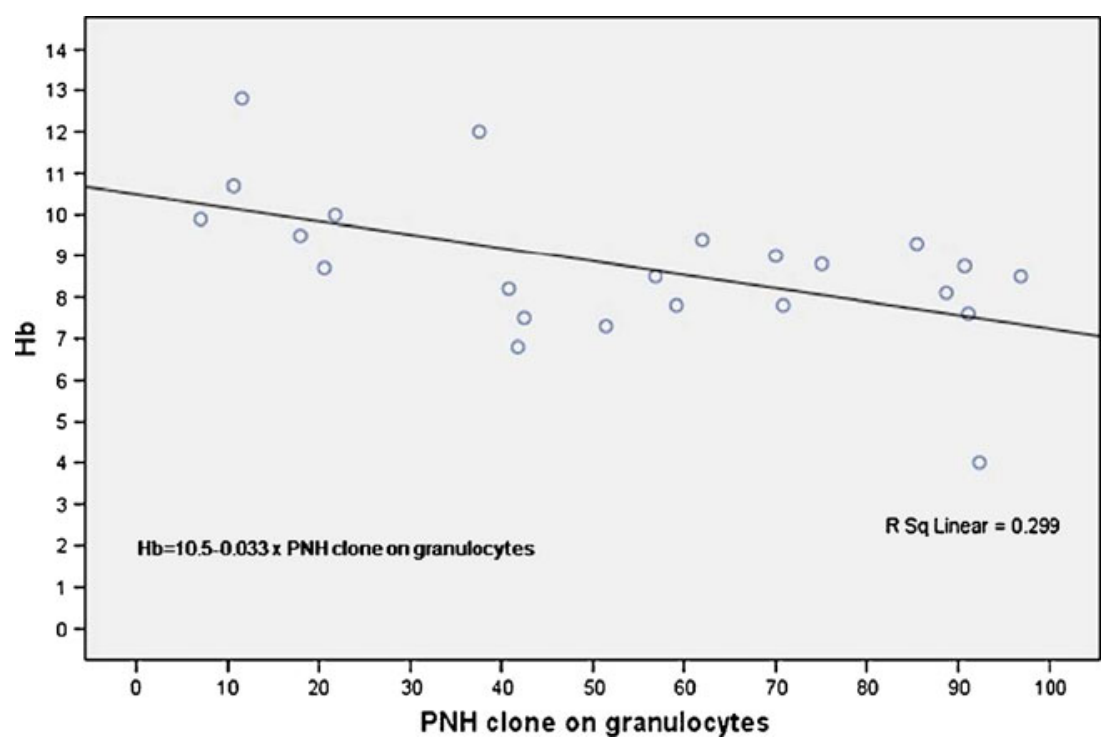

No significant correlation was seen between the PNH clone size on granulocytes and transfusion frequency $(p>$ $0.05)$. Similarly, no significant correlation was seen between the PNH clone size on granulocytes and reticulocyte count, platelet count, ANC, LDH, and plasma hemoglobin $(p>0.05)$. The patients are being followed up (1-60 months) and receiving regular transfusion support, androgens, immunosuppressants, corticosteroids, and hematinics either alone or in combination.

Patients with aplastic anemia

Forty-six patients $(30 \mathrm{M}, 16 \mathrm{~F})$ of aplastic anemia with median age of 27 (5-68)years had a median $\mathrm{Hb}$ of 8.4 (1.8$11.4) \mathrm{g} / \mathrm{dL}$, TLC $2.7(0.9-8) \times 10^{9} / \mathrm{L}$, ANC $0.8(0.04-6.8) \times$ $10^{9} / \mathrm{L}$, and platelet count $15(2-165) \times 10^{9} / \mathrm{L}$. Out of 46 aplastic anemia patients, PNH clone was detected in 23 $(50 \%)$ patients. On comparing patients of AA with $(n=23)$ and without $(n=23) \mathrm{PNH}$ clone, no significant difference was seen in $\mathrm{Hb}$, ANC, platelet count, and transfusion frequency in two groups (Table 2).

The clone size was $<1 \%$ in eight $(17.4 \%), 1-10 \%$ in nine $(19.6 \%)$, and $10-50 \%$ in six $(13 \%)$ patients. The transfusion frequency in patients varied from 15 days to 6 months
(Table 3). The PNH clone size was $0.4 \%$ to $38.7 \%$ on granulocytes and correlated with that on monocytes $(0.8 \%$ to $55.8 \% ; r=0.728 ; p<0.01)$. However, the PNH clone size on granulocytes did not correlate with the red cell transfusion frequency, $\mathrm{Hb}, \mathrm{ANC}$, or platelet count $(p>0.05)$.

Patients with myelodysplastic syndrome

Seven cases of MDS (6 M, $1 \mathrm{~F}$ ) included five cases of refractory anemia (RA) and two cases of refractory cytopenia with multilineage dysplasia (RCMD). Their median $\mathrm{Hb}$ was $6.5(3.2-9.5) \mathrm{g} / \mathrm{dL}$, platelet count $13(7-550) \times 10^{9} / \mathrm{L}$, TLC 2.6 $(2.1-9.7) \times 10^{9} / \mathrm{L}$, and ANC $1.1(0.4-6.3) \times 10^{9} / \mathrm{L}$. PNH clone $(<1 \%$ clone) was seen in three $(42.9 \%)$ patients (two RA, one RCMD) receiving transfusion every 2 to 4 months. No statistical analysis could be done due to small sample size in these patients.

\section{Discussion}

Flow cytometric analysis of granulocytes can be done using various staining methods like lyse-wash-stain technique, stain-lyse-wash technique, and stain-no lyse-no wash

Table 1 Distribution of PNH cases $(n=23)$ according to PNH clone size and transfusion requirement

\begin{tabular}{lcl}
\hline PNH clone size on granulocytes (\%) & No. of cases $(\%)$ & Median transfusion frequency in months (range) \\
\hline $1-10$ & $1(4.3)$ & 1 \\
$10-50$ & $6(26.1)$ & $6(1-13)$ \\
$>50$ & $16(69.6)$ & $4(0.5-15)$ \\
\hline
\end{tabular}


Table 2 Comparison of aplastic anemia cases without $(n=23)$ and with $(n=23)$ PNH clone

\begin{tabular}{lll}
\hline & $\begin{array}{l}\text { AA patients without PNH clone }(n=23) \\
\text { Median (range) }\end{array}$ & $\begin{array}{l}\text { AA patients with PNH clone }(n=23) \\
\text { Median (range) }\end{array}$ \\
\hline Age (years) & $35(13-68)$ & $20(5-55)$ \\
M/F ratio & $2.3: 1$ & $1.6: 1$ \\
Hemoglobin & $8.4(1.8-10.2)$ & $8.4(4.1-11.4)$ \\
Absolute neutrophil count $\left(\times 10^{9} / \mathrm{L}\right)$ & $1.06(0.1-4.4)$ & $0.5(0.04-6.8)$ \\
Platelet count $\left(\times 10^{9} / \mathrm{L}\right)$ & $15(4-91)$ & 0.66 \\
Transfusion frequency (months) & $2(0.5-6)$ & 0.085 \\
\hline
\end{tabular}

technique [1]. However, there is no consensus on the staining method, antibodies selected as well as gating strategy for analysis.

The CD55/CD59 combination using different fluorochromes, as used in this study, has the advantage of detecting the absence of both antigens on the same cell in comparison to staining done in separate tubes. In addition, this staining technique allows simultaneous analysis of monocytes in the same tube with CD45/SSC gating. Thus, demonstrating the lack of these two markers on two different cell lines (granulocytes and monocytes) increases the specificity of $\mathrm{PNH}$ diagnosis. The latest guidelines for PNH diagnosis, however, do not recommend CD55/CD59 combination as they may give less separation between positive and negative populations than other GPI-linked antigens [2]. Antibodies such as CD16, CD24, and CD66b have been reported to work equally well for granulocyte analysis. Combination of CD16/CD66b has the added advantage of clearly separating granulocytes from eosinophils and is shown to be superior to CD55/CD59 in screening for subclinical PNH [3].

The PNH clone size on granulocytes significantly correlated with that on monocytes $(r=0.563 ; p<0.01)$ in this study. Monocyte and granulocyte clone sizes correlate well with each other as they both are derived from a common progenitor. Although monocyte analysis for $\mathrm{PNH}$ clone may provide additional information, it may be technically difficult due to their low absolute number in patients with pancytopenia. Using CD45/SSC gating, we felt that monocytes were easier to gate than granulocytes as they tend to make a tighter cluster than scattered and markedly depleted granulocytes in these patients. Moreover, the analysis could be done in the same tube without requiring any additional reagents.

PNH clone size on granulocytes negatively correlated with the hemoglobin of patient in our study $(r=-0.523 ; p<$ 0.05). Earlier, Pramoonjago et al. [4] recorded negative correlation $(r=0.374)$ between $\mathrm{Hb}$ and CD59-negative granulocytes in $\mathrm{PNH}$ and another group documented even higher negative correlation coefficients $(r>0.80)$ between $\mathrm{Hb}$ and the percentage of GPI-AP-negative monocytes and granulocytes [5].

Thrombosis developed in two of our 23 patients $(8.6 \%)$ on follow-up. They had a PNH clone size of $70 \%$ and $88.7 \%$ on granulocytes and developed left leg DVT and right middle cerebral artery thrombosis, respectively. Two recent studies with relatively large number of patients have shown that those who have granulocyte $\mathrm{PNH}$ clones in excess of $50 \%$ are at a major risk of developing thrombosis $[6,7]$ In one of these series, 7.2\% PNH patients at presentation and $25.6 \%$ during follow-up developed thrombosis. These differences in the incidence of thrombosis may indicate that Indian patients have a lower risk of thrombosis compared to Western population.

The detection rate of PNH defect in bone marrow failure syndromes, namely aplastic anemia and MDS, has varied over the years, primarily due to the variable sensitivity and cutoffs of the tests employed. Flow cytometry can detect GPI anchordeficient cell populations in 29-52\% AA patients during follow-up [8]. Using a highly sensitive flow cytometry having a cutoff of $>0.003 \%$ granulocytes, the frequency of $\mathrm{PNH}$ granulocytes was reported to be as high as $88.6 \%$ of 35
Table 3 Distribution of aplastic anemia cases $(n=46)$ according to PNH clone size and transfusion requirement

\begin{tabular}{lcc}
\hline PNH clone size on granulocytes & No. of cases (\%) & Median transfusion frequency in months (range) \\
\hline No PNH clone & $23(50)$ & $2(0.5-6)$ \\
$<1 \%$ & $8(17.4)$ & $1(0.5-4)$ \\
$1-10 \%$ & $9(19.6)$ & $1.5(1-6)$ \\
$10-50 \%$ & $6(13)$ & $1(0.5-3)$ \\
\hline
\end{tabular}


patients of untreated AA at diagnosis [9]. We found a $\mathrm{PNH}$ clone in $23(50 \%)$ of our AA patients at diagnosis with the assay sensitivity being $>0.2 \%$ granulocytes.

Iwanaga et al. have detected PNH clone using FCM in $10 \%$ of 40 MDS patients [10]. However, none of the 20 MDS patients in another study showed PNH clone [11]. We found a minor PNH clone in three (two RA, one RCMD) of our seven MDS patients. These patients can be classified as $\mathrm{PNH}$ subclinical (PNH-sc) in the setting of another specified bone marrow disorder (i.e., PNH-sc/RA-MDS or PNH-sc/RCMD-MDS) according to a classification proposed by International PNH interest group (IPIG) [12]. This classification divides PNH into three groups, namely classic $\mathrm{PNH}, \mathrm{PNH}$ in the setting of another specified bone marrow disorder, and PNH subclinical in the setting of another specified bone marrow disorder.

Typically, fewer than 10\% GPI-AP-deficient granulocytes are detected in AA patients at diagnosis, but occasional patients may have larger clones [13]. Most of our AA patients showed absence of PNH clone or a minor $(<10 \%)$ PNH clone. These patients with a minor PNH clone can be labeled as PNH-sc/AA as per IPIG classification. However, it is not very clear whether the six AA patients with $>10 \%$ PNH clone should be classified as $\mathrm{PNH}$ in the setting of another specified bone marrow disorder (i.e., PNH/AA) or PNH-sc/AA in absence of any evidence of hemolysis. Moreover, the clinical significance of such a reclassification is yet to be ascertained during their workup for bone marrow transplant. The PNH clone size in these cases did not significantly correlate with parameters of disease severity. Detection of PNH clones in AA and MDS suggests a pathogenetic link to immunologically mediated forms of bone marrow failure. Previously, various groups have reported that $23-38 \%$ patients of PNH may have antecedent history of aplastic anemia [14]. Six out of 23 (26.1\%) PNH patients in our study had prior history of aplastic anemia, and the duration between diagnosis of AA and PNH was 1 to 5 years. Most (five out of 6; 83.3\%) of these patients developed hemolytic $\mathrm{PNH}$, and a 15 -year male AA patient had responded to anti-thymocyte globulin 2 years back, before relapsing with hemolytic features. One of the possible causes of expansion of PNH clone in AA patients may be that the PNH stem cells acquire survival advantages due to a deficiency of GPI-anchored proteins required for being attacked by cytotoxic T cells [15].

There have been conflicting reports about therapeutic significance of existing PNH clone in AA and MDS. Some studies have not shown any close relationship between the detection of PNH clone and the response to therapy, both in MDS [10] as well as in AA patients [16]. Dunn et al., however stated that the presence of GPI-anchored proteindeficient cells in MDS predicts responsiveness to immunosuppressive therapy as there was a significant correlation between the PNH population and hematologic improvement after anti-thymocyte globulin [17]. A higher probability of response to cyclosporine therapy was also shown in MDS (RA) patients with PNH clone (PNH + RA patients) compared to patients without increased $\mathrm{PNH}$ cells by Wang et al. [18]. The current study has a limited number of such patients to analyze the clinical significance of $\mathrm{PNH}$ clone in AA and MDS patients.

\section{Conclusion}

To conclude, flow cytometry is a sensitive and reliable method of PNH clone assessment in PNH and other bone marrow failure syndromes. Despite the availability of many markers of PNH, CD55 and CD59 have the advantage of being simultaneously applied to granulocytes and monocytes for PNH assessment. The CD55/CD59 combination using different fluorochromes in the same tube detects cells with absence of both antigens, i.e., the true PNH clone. This increases the sensitivity of the assay compared to staining done in separate tubes. Although $\mathrm{PNH}$ clone correlated with the degree of anemia in $\mathrm{PNH}$ patients, a larger patient dataset needs to be assessed to conclusively prove the impact of PNH clone of variable size on parameters of clinical severity in AA and MDS patients.

Acknowledgments The financial assistance for the project was provided by the Indian Council Of Medical Research under its "Financial assistance to DM-thesis" program. We thank Dr. Guresh Kumar for statistical assistance. We also thank Mrs. Satyawati Yadav, Mr. Rahul Sharma, and Mr. Nemat for their help in the sample processing.

Conflict of interest The authors have no conflict of interest to declare.

\section{References}

1. Hernández-Campo PM, Martín-Ayuso M, Almeida J et al (2002) Comparative analysis of different flow cytometry-based immunophenotypic methods for the analysis of CD59 and CD55 expression on major peripheral blood cell subsets. Cytometry B Clin Cytom 50:191-201

2. Borowitz MJ, Craig FE, Digiuseppe JA et al (2010) Guidelines for the diagnosis and monitoring of paroxysmal nocturnal hemoglobinuria and related disorders by flow cytometry. Cytometry B Clin Cytom 78:211-230

3. Wang SA, Pozdnyakova O, Jorgensen JL et al (2009) Detection of paroxysmal nocturnal hemoglobinuria clones in patients with myelodysplastic syndromes and related bone marrow diseases, with emphasis on diagnostic pitfalls and caveats. Haematologica 94:29-37

4. Pramoonjago P, Pakdeesuwan K, Siripanyaphinyo U et al (1999) Genotypic, immunophenotypic and clinical features of Thai patients with paroxysmal nocturnal hemoglobinuria. Br J Haematol 105:497-504 
5. Piedras J, Lopez-Karpovitch X (2000) Flow cytometric analysis of glycosylphosphatidyl inositol-anchored proteins to assess paroxysmal nocturnal hemoglobinuria clone size. Cytometry 42(4):234-238

6. Moyo VM, Mukhina GL, Garrett ES et al (2004) Natural history of paroxysmal nocturnal haemoglobinuria using modern diagnostic assays. Br J Haematol 126:133-138

7. de Latour RP, Mary JY, Salanoubat C et al (2008) Paroxysmal nocturnal hemoglobinuria: natural history of disease subcategories. Blood 112:3099-3106

8. Nishimura J, Murakami Y, Kinoshita T (1999) Paroxysmal nocturnal hemoglobinuria: an acquired genetic disease. Am J Hematol 62:175-182

9. Wang H, Chuhjo T, Yamazaki H et al (2001) Relative increase of granulocytes with a paroxysmal nocturnal hemoglobinuria phenotype in aplastic anaemia patients: the high prevalence at diagnosis. Eur J Haematol 66:200-205

10. Iwanaga M, Furukawa K, Amenomori T et al (1998) Paroxysmal nocturnal haemoglobinuria clones in patients with myelodysplastic syndromes. Br J Haematol 102:465-474

11. Varma N, Varma S, Vohra H (2000) Flowcytometric detection of PNH defect in Indian patients with aplastic anemia and myelodysplastic syndromes. Am J Hematol 65(3):264-265
12. Parker C, Omine M, Richards S et al (2005) Diagnosis and management of paroxysmal nocturnal hemoglobinuria. Blood 106:3699-3709

13. Mukhina GL, Buckley JT, Barber JP et al (2001) Multilineage glycosylphosphatidylinositol anchor deficient hematopoiesis in untreated aplastic anemia. Br J Haematol 115:476-482

14. Parker CJ (2009) Bone marrow failure syndromes: paroxysmal nocturnal hemoglobinuria. Hematol Oncol Clin N Am 23:336-346

15. Takami A, Zeng W, Wang H, Matsuda T, Nakao S (1999) Cytotoxicity against lymphoblastoid cells mediated by a T-cell clone from an aplastic anaemia patient: role of CD59 on target cells. Br J Haematol 107:791-796

16. DeLord C, Tooze JA, Saso R et al (1998) Deficiency of glycosylphosphatidyl inositol-anchored proteins in patients with aplastic anaemia does not affect response to immunosuppressive therapy. Br J Haematol 101:90-93

17. Dunn DE, Tanawattanacharoen P, Boccuni P et al (1999) Paroxysmal nocturnal hemoglobinuria cells in patients with bone marrow failure syndromes. Ann Intern Med 131:401-408

18. Wang H, Chuchjo T, Yasue S et al (2002) Clinical significance of a minor population of paroxysmal nocturnal hemoglobinuria-type cells in bone marrow failure syndrome. Blood 100:3897-3902 